\title{
Making sense of our pain. A group intervention in patients diagnosed with dysthymia and fibromyalgia.
}

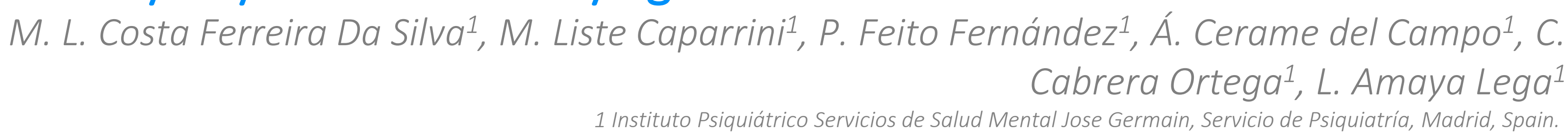

\section{OBJETCTIVE}

We present a descriptive analysis of the results of a psychotherapeutic intervention in patients with concomitant diagnoses of Persistent Depressive Disorder (PPD), also known as Dysthymia, and fibromyalgia.

\section{BACKGROUND AND AIMS}

Dysthymia is an increasingly prevalent diagnostic challenge in the Spanish clinical context. This diagnostic entity has a chronic course and tends to be resistant to pharmacological treatment. Moreover, negative countertransference and psychosomatic symptoms make psychotherapeutic treatment difficult by invading the patient's discourse insofar as patients tend to emphasise the description of pain and the feeling of neglect to which they feel subjected by the social environment and medical professionals.

\section{MATERIALS AND METHODS}

We study a 9-month psychodynamic group intervention. Selection criteria include people whose clinical presentation is likely to fall under the diagnostic category of Persistent Depressive Disorder. Until the creation of the group, these patients have usually undergone individual psychotherapy in the context of outpatient-Mental Health services. Even though it is not chosen as criteria, all patients selected for group treatment are female and have a concomitant diagnosis of fibromyalgia.

The results of pre and post-intervention tests are analysed by comparing individual discrete values and the total averages. Scales used: Beck Depressive Inventory (BDI), Social Adaptation Self-evaluation Scale (SASS), Fibromyalgia Impact Questionnaire (FIQ) and Clinical Anxiety Scale (CAS).

\section{PRE AND POST INTERVENTION AVERAGES IN SCALE RESULTS}

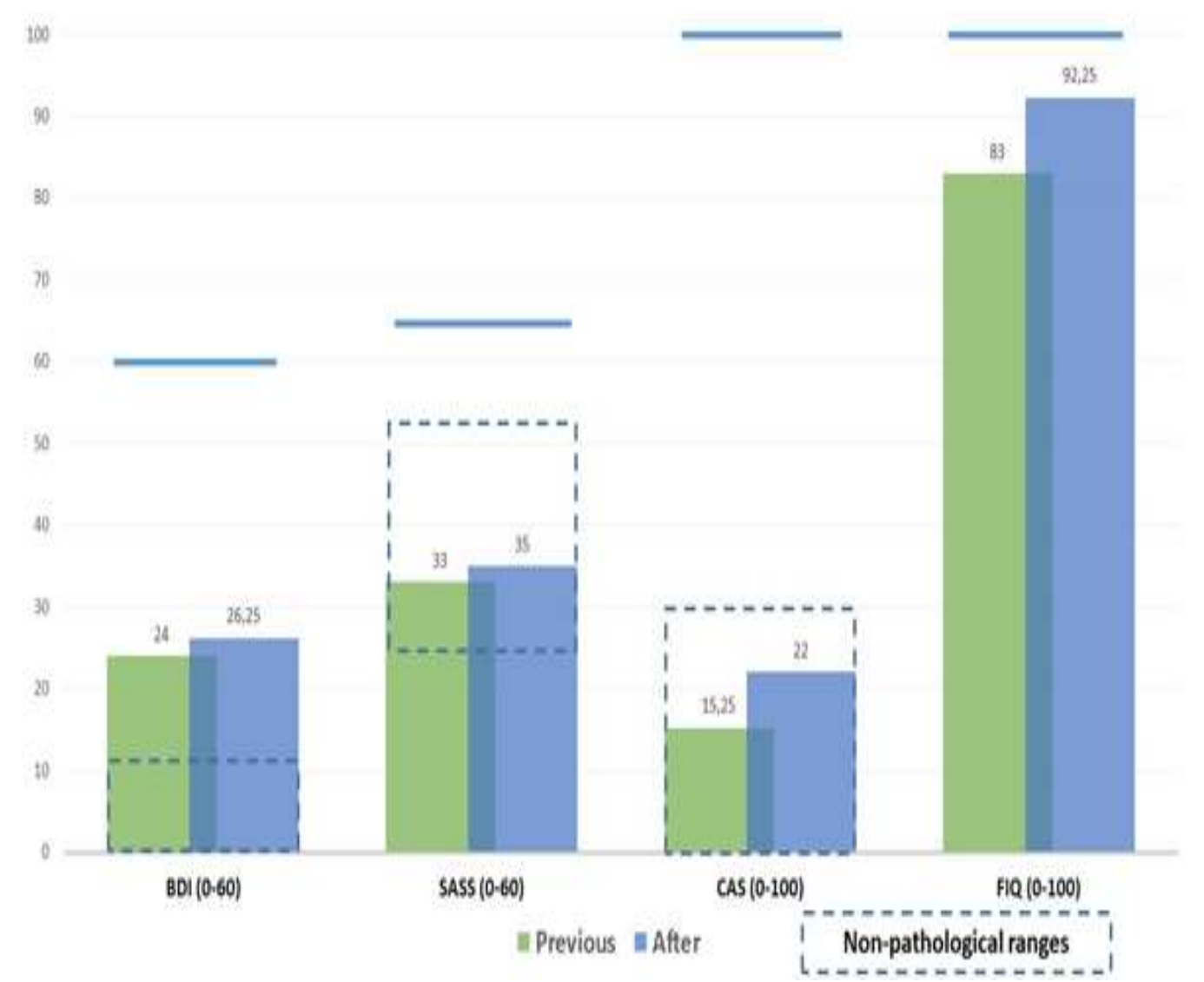

\section{DISCUSSION}

The differences in averages are of a slight worsening in the BDI, CAS and FIQ scales. However, we can find a slight improvement in the SASS test, suggesting a better social adaptation of patients.

The average of pre and post intervention scores found on the $\mathrm{BDI}$ scale are within pathological ranges indicating the presence of depressive symptomatology. These results are consistent with the clinical presentation of the patients in individual interventions. In contrast, all pre and post treatment results are found in non-pathological ranges of CAS and SASS scales, indicating an adequate social adaptation of patients to their environments despite the subjective impression transmitted by them. The pre and post intervention results on the FIQ scale are strikingly high.

\section{CONCLUSIONS}

Several considerations arise from the analysis of the information obtained through the study of patients included in the group. Firstly, the results of the BDI test expose the amount of psychological distress these patients suffer from. Patients presented a depressive mood which, counterintuitively, did not limit their social adaptation, according to the results obtained in the SASS test. These results suggest that patients with PPD express their psychological distress in a different manner compared to other depressed individuals. This particular form of expression of distress can be a source of the misunderstanding and distrust from their social environments and attending physicians. In this atypical presentation of mood disturbances, physical suffering takes on a major role. Particularly high pre and post-intervention values in the FIQ test can be interpreted as the expression of intense psychological distress through pain. During group therapy, participants found a connection between the intensification of their pain and the vital events that cause them suffering in the first place.

It is hypothesized that the reduction found in some of the scales is due to an improved contact of patients with their psychic as well as physical suffering resulting from a deep psychotherapeutic effort hitherto impossible due to the imperviousness of this type of patients. What is more, it is believed that this contact could determine a change towards a consistent and lasting improvement. Therefore, it is considered that the intervention had also provided benefits for patients. However, given the limitations this study presents, it is necessary to further our understanding through the analysis of more group interventions in patients diagnosed with PPD and fibromyalgia. 